\title{
Efeito do Fotoperíodo sobre o Desenvolvimento de Girinos de Rã-touro (Rana catesbeiana Shaw, 1802) ${ }^{1}$
}

\author{
Andrea Cecchetto Bambozzi ${ }^{2}$, José Teixeira de Seixas Filho ${ }^{3}$, Luciana Almada Thomaz ${ }^{4}$, \\ Lídia Miyako Yoshii Oshiro 5
}

\begin{abstract}
RESUMO - O objetivo deste trabalho foi avaliar o efeito de diferentes fotoperíodos sobre o peso, comprimento e a metamorfose de girinos de rã-touro ( Rana catesbeiana Shaw, 1802). Foram utilizados 720 animais de uma mesma desova, no estágio 25, segundo Gosner (1960), distribuídos em 24 caixas de polietileno, com 30 litros de volume d'água, resultando em uma densidade de 1 girino por litro. Os tratamentos consistiram em seis fotoperíodos [0L:24D; 8L:16D; 12L:12D; 14L:10D (natural, como testemunho); 16L:8D e 24L:0D] e quatro repetições, sob temperatura ambiente, sendo observados durante os meses de dezembro de 2000 a abril de 2001 . O fotoperíodo foi programado utilizando-se cobertura das caixas com plástico preto, exceto para o fotoperíodo natural (plástico incolor), e três programadores para as lâmpadas fluorescentes de 21 watts referentes aos fotoperíodos 8L:16D, 16L:8D e 12L:12D. O delineamento experimental foi inteiramente casualizado em esquema de parcelas subdivididas split-plot (coletas no tempo). O experimento foi analisado, utilizando-se o melhor modelo de regressão. Os girinos apresentaram ganhos de comprimento e de peso com tendência cúbica para maioria dos tratamentos, exceto para o comprimento referente ao fotoperíodo 24L:0D, que apresentou tendência quadrática. Para avaliar a metamorfose, foram realizadas análises estatísticas pela ANOVA (Análise de Variância) e teste de Duncan, em nível de 5\%. O melhor tratamento deu-se com os animais submetidos ao fotoperíodo 12L:12D, aos 120 dias, que apresentaram o maior peso (1,80 g) e melhor taxa final de metamorfose, com cerca de $50 \%$ da população neste processo. O pior resultado foi obtido com os animais que receberam 24L:0D de fotoperíodo, apresentando aos 135 dias maior peso (2,0 g), com 4\% de taxa metamórfica. Este resultado demonstrou que este fotoperíodo inibiu o desenvolvimento do girino. O pior resultado seguinte foi obtido para o tratamento 16L:8D. Os tratamentos 0L:24D, 8L:16D e natural apresentaram resultados intermediários quanto à metamorfose.
\end{abstract}

Palavras-chave: crescimento, fotoperíodo, girino, metamorfose, Rana catesbeiana, rã-touro

\section{Effect of the Photoperiod on Bullfrog (Rana catesbeiana Shaw, 1802) Tadpoles Development}

ABSTRACT - The objective of this work was to evaluate the performance of the bullfrog tadpoles (Rana catesbeiana Shaw, 1802) under different photoperiods. Seven hundred and twenty animals from the same spawn were used, on the stage 25 , according to Gosner (1960). The fishes were allotted to 24 polyethylene $30 \mathrm{~L}$ boxes, with a density of one tadpole per liter. The treatments consisted of six photoperiods [0L:24D; 8L:16D; 12L:12D; 14L:10D (natural as witnessed); 16L:8D and 24L:0D] and four replicates, using environment temperature. The experiment was developed from December 2000 to April 2001. The photoperiods were programmed using the black plastic covering the boxes, except for natural photoperiod (colorless plastic). Three timers with 21 watts fluorescent lamps, to photoperiods of $8 \mathrm{~L}: 16 \mathrm{D} ; 16 \mathrm{~L}: 8 \mathrm{D}$ and $12 \mathrm{~L}: 12 \mathrm{D}$, were also used. The statistical analyses were performed by a complete randomized split-plot design. The experiment was analyzed managing best regression model. Tadpoles presented length and weight gain with cubic tendency for most of the treatments, except for length for 24L:0D photoperiod, that showed quadractic tendency. For metamorphosis evaluation, statistical analyses were performed by ANOVA and Duncan test at $5 \%$ level. The best result was obtained in a 12L:12D photoperiod, as in 120 days the bullfrog tadpoles showed the greatest weight $(1.80 \mathrm{~g})$ and the best metamorphic rate with about $50 \%$ of the population on this process. After 120 days, the animals showed smaller weight, that characterized the complete metamorphic process. The worst result occurred in the 24L:0D photoperiod. Higher weight $(2.0 \mathrm{~g})$ was obtained with $4 \%$ of metamorphic rate. This result showed that the photoperiod inhibited the development of tadpoles. The following worst result was obtained for the treatment $16 \mathrm{~L}: 8 \mathrm{D}$. The photoperiods $0 \mathrm{~L}: 24 \mathrm{D}, 8 \mathrm{~L}: 16 \mathrm{D}$ and the natural process showed the intermediate results.

Key Words: bullfrog, growth, metamorphosis, photoperiod, Rana catesbeiana, tadpoles

\footnotetext{
1 Parte da Dissertação de Mestrado em Zootecnia apresentada pelo primeiro autor à UFRRJ financiada pela FAPERJ.

2 Aluna de Mestrado do Instituto de Zootecnia da UFRRJ. E.mail: abambozzi@yahoo.com.br

3 Pesquisador da FIPERJ. E.mail: joseseixas@fiperj.rj.gov.br

${ }^{4}$ Aluna de mestrado do IZ - UFRRJ. E.mail: almadalt@yahoo.com.br

${ }^{5}$ Professora Adjunto do IZ (DPA) da UFRRJ. E.mail: oshiro@ufrrj.br
} 


\section{Introdução}

Poucos estudos têm sido realizados para testar a influência dos fatores ambientais sobre o desenvolvimento larval e metamorfose em anfíbios (Delgado et al., 1987). Entre estes fatores, a luz é um dos mais difíceis de ser estudado. O desenvolvimento das larvas de anfíbios é extremamente variável (Rose, 1960; Wilbur \& Collins, 1973; Gromko et al., 1973), mesmo quando criadas sob condições bem controladas de laboratório, os dados obtidos não são facilmente interpretados (Culley Jr. et al., 1977).

Entre as dificuldades para a criação de girinos, relata-se o pouco conhecimento sobre a exigência nutricional, a temperatura e o fotoperíodo necessários ao ótimo desempenho animal.

O fotoperíodo representa um papel importante na regulação da taxa da metamorfose (Dodd \& Dodd, 1976) e, segundo Hoserman et al. (1976), variáveis como fotoperíodo e manejo inadequado podem provocar distúrbios que alteram a resposta dos girinos, com relação ao ganho de peso e à taxa de metamorfose. Culley \& Sotiaridis (1983) relataram que outros fatores, além do fotoperíodo, também podem afetar a sobrevivência, o desenvolvimento e a taxa de metamorfose destes animais, como densidade, qualidade da água e características físicas do alimento.

Segundo Delgado et al. (1984) e Gutierrez et al. (1984), a fase longa de luz inibiu o desenvolvimento de Discoglossus pictus e Xenopus laevis, entretanto, em girinos do gênero Rana o desenvolvimento foi mais rápido (Delgado et al., 1987). Filadelfi \& Castrucci (1996) verificaram que a escuridão contínua inibiu o crescimento e desenvolvimento de girinos de Rana esculenta e Rana temporaria, enquanto o fotoperíodo curto aumentou o ganho de peso e estimulou a metamorfose em larvas de $X$. laevis.

O objetivo deste estudo foi avaliar a melhor taxa de metamorfose com o maior ganho de peso e comprimento em girinos de rã-touro, utilizando seis fotoperíodos.

\section{Material e Métodos}

O experimento foi desenvolvido no Laboratório da Estação de Aquicultura Almirante Paulo Moreira (EAAPM) da Fundação Instituto de Pesca do Estado do Rio de Janeiro (FIPERJ), tendo início em dezembro de 2000 e término em abril de 2001, perfazendo um total de 135 dias de experimentação.
Foram adquiridos 720 girinos de rã-touro (Rana catesbeiana Shaw, 1802), provenientes de uma mesma desova, ainda na fase larval, sendo acondicionados no laboratório até atingirem o estágio 25 , segundo Gosner (1960). As unidades experimentais foram dispostas lado a lado sobre a bancada. Os girinos foram distribuídos em 24 caixas de polietileno $(62,5 \times 40 \times 16 \mathrm{~cm})$, com capacidade para 40 litros, de coloração amarela, as quais receberam $30 \mathrm{~L}$ de água, acondicionando 30 animais com peso e comprimento médio inicial de $0,017 \pm 0,004 \mathrm{~g}$ e $3,73 \pm 0,145 \mathrm{~mm}$, respectivamente, resultando em uma densidade de 1 girino por litro.

A renovação da água nas caixas foi aproximadamente de $200 \%$ do volume a cada 24 horas, com abastecimento individual e sistema de escoamento do tipo "joelho", com saída da água de fundo, assim como o controle do volume.

A água utilizada foi proveniente da Companhia Estadual de Água e Esgoto (CEDAE) e o cloro foi eliminado por meio de 4 filtros de carvão ativado compostos por cano PVC (Ø $100 \mathrm{~mm}$ ) e lã de vidro, nas suas extremidades, superior e inferior. Cada unidade experimental recebeu aeração constante, por meio de soprador e mangueiras plásticas 3/16", providas de pedras porosas em suas extremidades e regulada por registro de mesmo calibre.

O fotoperíodo foi programado utilizando-se cobertura das caixas com plástico preto tipo "terreiro", exceto para o fotoperíodo natural (plástico incolor), e lâmpadas fluorescentes de 21 watts. O controle foi efetuado por meio de programadores, tipo "timer", referentes aos fotoperíodos 8L:16D, 16L:8D e 12L:12D, respectivamente. Já as lâmpadas referentes ao fotoperíodo 24L:0D permaneceram ligadas 24 horas durante todo o período experimental.

Os animais foram alimentados, ad libitum, com ração comercial farelada contendo $45 \% \mathrm{~PB}$, duas vezes ao dia, às $9 \mathrm{~h} 30 \mathrm{e} 14 \mathrm{~h} 30$. A limpeza das caixas foi realizada, diariamente, pela manhã $(8 \mathrm{~h})$, por sifonagem de fundo, retirando-se as fezes e os restos alimentares.

Diariamente, pela manhã e tarde, foram tomadas as temperaturas do ar e da água por meio de termômetro de coluna de mercúrio, com escala em centígrados, de 0 a $60^{\circ} \mathrm{C}$. O controle da amônia e do $\mathrm{pH}$ foi efetuado uma e duas vezes, respectivamente, a cada semana, por meio de kit utilizado para controle de água de piscina.

As biometrias foram realizadas quinzenalmente, avaliando-se ganho de peso, comprimento e sobrevivência. Os girinos foram colocados sobre toalha de 
pano umedecida, para retirada do excesso de água, sem que houvesse desidratação abrupta e mensurados da boca até a inserção da cauda, com auxílio de paquímetro digital, com precisão de centésimos de milímetro. A seguir, cada animal foi transferido para recipiente plástico com volume de aproximadamente $5 \mathrm{~mL}$ de água e pesado em balança analítica, com precisão de $0,001 \mathrm{~g}$ (previamente tarado). A obtenção do número total de imagos, no clímax da metamorfose, estágio 43, segundo Gosner (1960), foi realizado por meio de coleta à medida que ocorria o surgimento das patas anteriores, sendo retirados do experimento e anotados.

O delineamento experimental foi inteiramente casualizado, com seis tratamentos [fotoperíodos 0L:24D; 8L:16D; 12L:12D; 14L:10D (natural como testemunho); 16L:8D e 24L:0D] e quatro repetições, em um esquema em parcelas subdivididas, sendo as subparcelas as biometrias no tempo.

A distribuição dos tratamentos no módulo experimental foi realizada ao acaso, por meio de sorteio das caixas. Os animais foram avaliados em dez biometrias, realizadas quinzenalmente até o fim do período experimental. Para avaliar a metamorfose, foram realizadas análises estatísticas pela ANOVA e pelo teste Duncan, em nível de 5\%.

As análises estatísticas foram feitas com o programa SAEG (Sistema de Análises Estatísticas e Genéticas), desenvolvido por Euclydes (1983).

O modelo estatístico utilizado foi:

$$
Y_{i j k}=M+T_{i}+e_{i j}+(B / T)_{j}+(B * T)_{i j}+e_{i j k}
$$

em que $Y_{i j k}=$ observação referente ao fotoperíodo i, da biometria $\mathrm{j}$, na repetição $\mathrm{k} ; \mathrm{M}=$ efeito médio geral; $\mathrm{T}_{\mathrm{i}}=$ efeito do fotoperíodo, $\mathrm{i}=0,8,12,14,16,24$; $\mathrm{e}_{\mathrm{ij}}=$ erro aleatório da parcela no fotoperíodo $\mathrm{i}$ da biometria $\mathrm{j} ;(\mathrm{B} / \mathrm{T})_{\mathrm{j}}=$ efeito da biometria $\mathrm{j} ;(\mathrm{B} * \mathrm{~T})_{\mathrm{ij}}=$ efeito da interação fotoperíodo i e biometria $\mathrm{j} ; \mathrm{e}_{\mathrm{ijk}}=$ erro aleatório da subparcela no fotoperíodo i, da biometria $\mathrm{j}$, na repetição $\mathrm{k}$.

\section{Resultados e Discussão}

A temperatura do ar apresentou média de $31^{\circ} \mathrm{C}$ durante todo o experimento, e as médias da temperatura da água observadas pela manhã e pela tarde, foram, respectivamente, de 27 e $29^{\circ} \mathrm{C}$, exceto para o fotoperíodo natural, que apresentou no período da tarde média de $30^{\circ} \mathrm{C}$.
A média dos valores de $\mathrm{pH}$ e amônia foram, respectivamente, 7,4 e 0 . Tais resultados encontram-se dentro dos padrões considerados ótimos para a criação (Fontanello et al., 1982), demonstrando o bom funcionamento do sistema, no que se refere à taxa de renovação da água para os girinos de $200 \%$ ao dia, assemelhando-se ao trabalho desenvolvido por Seixas Filho et al. (1998).

A sobrevivência em 135 dias de experimento foi de $100 \%$ em todos os tratamentos. Os resultados obtidos ao final do experimento, em relação à média de comprimento e de peso dos girinos para cada fotoperíodo em ordem crescente, foram $21,90 \mathrm{~mm}$ e 1,44 g (0L:24D); 23,0 mm e 1,50 g (14L:10D natural); 23,33 mm e 1,78 g (8L:16D); 24,0 mm e 1,80 g (12L:12D); 23,90 mm e 1,90 g (16L:8D) e 25,0 mm e 2,0 g (24L:0D) (Figuras 1 e 2).

Pode-se observar que, inicialmente, os animais eram homogêneos, uma vez que não houve diferença significativa $(\mathrm{P}>0,05)$ no peso e no comprimento dos girinos distribuídos nos diferentes tratamentos.

$\mathrm{O}$ efeito dos tratamentos sobre as médias do comprimento e do peso nos girinos mostrou que houve diferença significativa $(\mathrm{P}<0,01)$ entre os fotoperíodos ao término do experimento (Tabelas 1e 2). Os girinos apresentaram ganho de comprimento e de peso com tendência cúbica para a maioria dos tratamentos, exceto para o comprimento referente ao fotoperíodo 24L:0D, que apresentou tendência quadrática (Tabela 1), o que pode ser confirmado pelas equações de regressão nas Tabelas 1 e 2 .

Os girinos mostraram uma característica própria de crescimento, que é o aumento do peso até o início da reabsorção da cauda no processo da metamorfose, seguido da diminuição no peso até transformarem-se em imagos. Estas observações estão de acordo com Hoffmann et al. (1989).

Os resultados apresentaram diferenças significativas $(\mathrm{P}<0,05)$, nos diferentes fotoperíodos para a metamorfose (Tabela 3).

O melhor resultado referente aos objetivos deste trabalho foi alcançado pelos girinos submetidos ao fotoperíodo 24L:0D, uma vez que atingiram $25 \mathrm{~mm}$ e 2,0 g de comprimento e peso, respectivamente, sendo os maiores parâmetros zootécnicos do experimento. Por outro lado, em relação à metamorfose, apresentou o pior resultado, com apenas $4,4 \%$ dos animais atingindo o estágio 43, segundo Gosner (1960). Esta variação de desenvolvimento dos girinos, mesmo sob condições iguais, são semelhantes às encontradas 


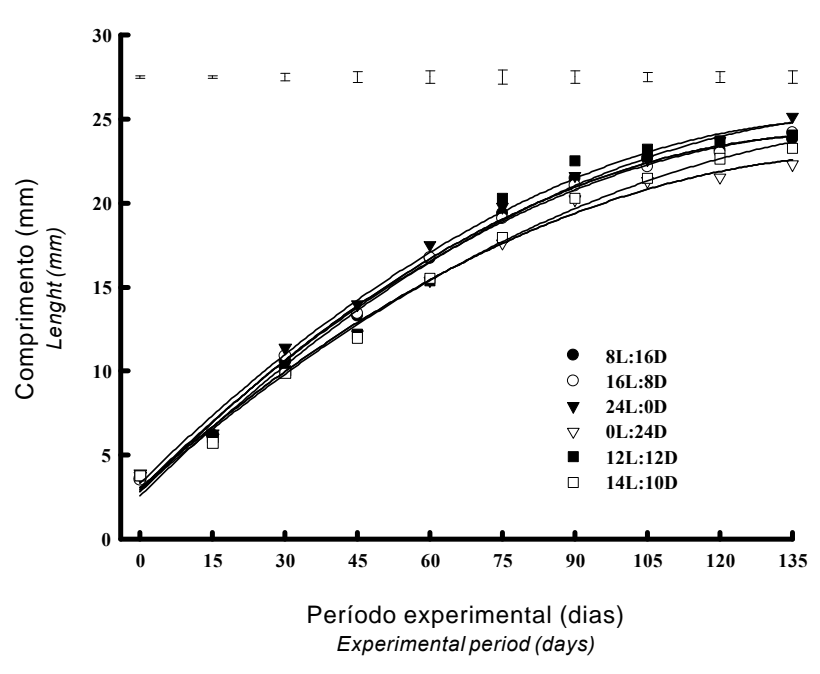

Figura 1 - Comprimento dos girinos de rã-touro (Rana catesbeiana), aos 135 dias, submetidos a diferentes fotoperíodos.

Figure 1 - Length of the bullfrog tadpoles (Rana catesbeiana), submitted to different photoperiods, within 135 days.

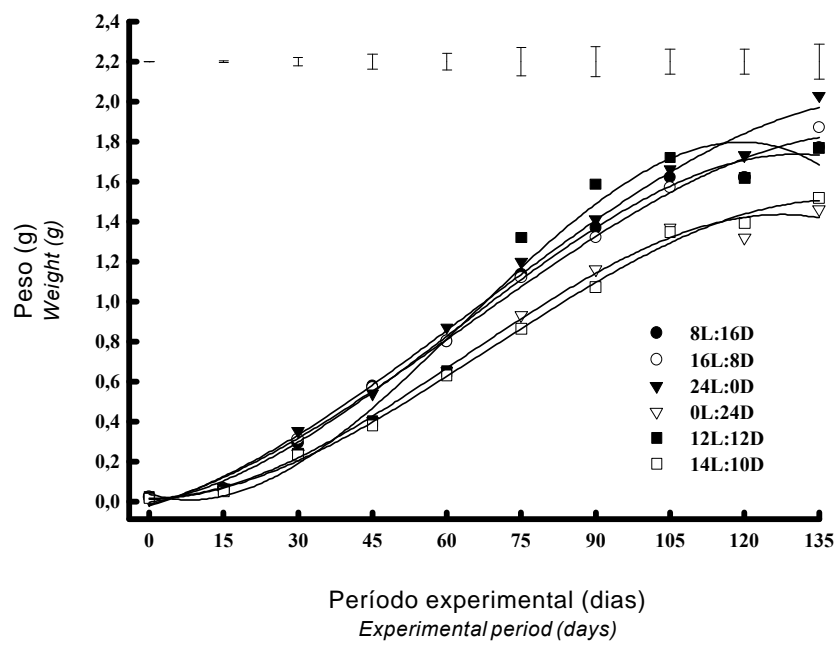

Figura 2 - Peso dos girinos de rã-touro (Rana catesbeiana), aos 135 dias, submetidos a diferentes fotoperíodos.

Figure 2 - Weight of the bullfrog tadpoles (Rana catesbeiana), submitted to different photoperiods within 135 days.

Tabela 1 - Quadrado médio da análise de variância do comprimento padrão dos girinos (CPAD), em função dos diferentes fotoperíodos

Table 1 - Average square of the analysis of variance of the standard length of tadpoles (CPAD), in function of the different fotoperiods

\begin{tabular}{|c|c|c|c|c|c|c|c|}
\hline \multirow{2}{*}{$\begin{array}{l}\text { Variável } \\
\text { Variable }\end{array}$} & \multirow{2}{*}{$\begin{array}{l}\text { G } \\
D F\end{array}$} & \multicolumn{6}{|c|}{$\begin{array}{l}\text { QM } \\
S M\end{array}$} \\
\hline & & $0 \mathrm{~L}^{1}$ & $8 \mathrm{~L}^{2}$ & $12 \mathrm{~L}^{3}$ & $14 \mathrm{~L}^{4}$ & $16 \mathrm{~L}^{5}$ & $24 \mathrm{~L}^{6}$ \\
\hline CPAD & 3 & $135,70 * *$ & $160,985 * *$ & $177,274 * *$ & $150,544 * *$ & $157,632 * *$ & \\
\hline \multirow[t]{3}{*}{$P A D C$} & 6 & 188 & 173 & 607 & 1996 & 245 & \\
\hline & 2 & & & & & & $247,274 * *$ \\
\hline & 7 & & & & & & 364 \\
\hline
\end{tabular}

$\mathrm{CV}=7.414$

$* *=(P<0,01)$

1 Efeito cúbico (cubic effect) $\quad \hat{Y}=3,59237+0,190379 X+4,51 \times 10^{-4} X^{2}-6,33 \times 10^{-6} X^{3} ; R^{2}=99,6$

2 Efeito cúbico (cubic effect) $\quad \hat{Y}=3,29583+2,335 \times 10^{-1} X+1,238 \times 10^{-4} X^{2}-5,515 \times 10^{-6} X^{3} ; R^{2}=99,7$

3 Efeito cúbico (cubic effect) $\quad \hat{Y}=3,71140+1,463 \times 10^{-1} X+1,831 \times 10^{-3} X^{2}-1,349 \times 10^{-5} X^{3} ; R^{2}=99$

4 Efeito cúbico (cubic effect) $\quad \hat{Y}=3,48537+1,852 \times 10^{-1} X+5,642 \times 10^{-4} X^{2}-6,370 \times 10^{-6} X^{3} ; R^{2}=99,6$

5 Efeito cúbico (cubic effect) $\quad \hat{Y}=3,12165+2,664 \times 10^{-1} X-6,341 \times 10^{-4} X^{2}-1,486 \times 10^{-6} X^{3} ; R^{2}=99,5$

${ }^{6}$ E feito quadrático (quadratic effect) $\hat{Y}=3,30962+2,855 \times 10^{-1} X-9,347 \times 10^{-4} X^{2} ; R^{2}=99,3$ 
Tabela 2 - Quadrado médio da análise de variância do peso dos girinos, em função dos diferentes fotoperíodos

Table 2 - Mean square of the analysis of variance of tadpoles weight, in function of the different photoperiods

\begin{tabular}{|c|c|c|c|c|c|c|c|}
\hline \multirow{2}{*}{$\begin{array}{l}\text { Variável } \\
\text { Variable }\end{array}$} & \multirow{2}{*}{$\begin{array}{l}\mathbf{G} \\
D F\end{array}$} & \multicolumn{6}{|c|}{$\begin{array}{l}\mathrm{QM} \\
S M\end{array}$} \\
\hline & & $0 \mathrm{~L}^{1}$ & $8 \mathrm{~L}^{2}$ & $12 \mathrm{~L}^{3}$ & $14 \mathrm{~L}^{4}$ & $16 \mathrm{~L}^{5}$ & $24 \mathrm{~L}^{6}$ \\
\hline Peso & 3 & $937 * *$ & $1,335 * *$ & $1,571 * *$ & $974 * *$ & $1,354 * *$ & $1,559 * *$ \\
\hline Weight & $\begin{array}{l}6 \\
2 \\
7\end{array}$ & 003 & 0027 & 018 & $\begin{array}{l}001 \\
247,274 * * \\
364\end{array}$ & 003 & 004 \\
\hline
\end{tabular}

$\mathrm{CV}=7,414$.

${ }^{* *}=(\mathrm{P}<0,01)$.

${ }^{1}$ Efeito cúbico (cubic effect) $\hat{Y}=1,036 \times 10^{-2}+3,441 \times 10^{-4} X+2,576 \times 10^{-4} X^{2}-1,354 \times 10^{-6} X^{3} ; R^{2}=99$

2 Efeito cúbico (cubic effect) $\hat{Y}=-6,002 \times 10^{-3}+3,843 \times 10^{-3} X+2,475 \times 10^{-4} X^{2}-1,339 \times 10^{-6} X^{3} ; R^{2}=99,4$

3 Efeito cúbico (cubic effect) $\hat{Y}=3,285 \times 10^{-2}-7,419 \times 10^{-3} X+4,952 \times 10^{-4} X^{2}-2,590 \times 10^{-6} X^{3} ; R^{2}=96,6$

4 Efeito cúbico (cubic effect) $\hat{Y}=9,735 \times 10^{-3}+6,730 \times 10^{-4} X+2,254 \times 10^{-4} X^{2}-1,099 \times 10^{-6} X^{3} ; R^{2}=99,6$

5 Efeito cúbico (cubic effect) $\hat{Y}=-2,304 \times 10^{-2}+7,037 \times 10^{-3} X+1,662 \times 10^{-4} X^{2}-8,683 \times 10^{-7} X^{3} ; R^{2}=99,3$

${ }^{6}$ E feito cúbico (cubic effect) $\hat{Y}=-2,2151 \times 10^{-2}+7,499 \times 10^{-3} X+1,699 \times 10^{-4} X^{2}-8,613 \times 10^{-7} X^{3} ; R^{2}=99,2$

por Rose (1960), Wilbur \& Collins (1973) e Gromko et al. (1973). Portanto, novos estudos tornam-se necessários para verificação da possibilidade de se reverter este quadro, viabilizando a obtenção de imagos de grande porte, uma vez que Culley Jr. et al. (1977) já haviam relatado as dificuldades de interpretação na criação destes animais.

Em situação intermediária em relação ao parâmetro zootécnico, foram apresentados os girinos submetidos aos fotoperíodos 8L:16D $(23,33 \mathrm{~mm}$ e $1,78 \mathrm{~g}), 12 \mathrm{~L}: 12 \mathrm{D}(24,0 \mathrm{~mm}$ e $1,80 \mathrm{~g})$ e $16 \mathrm{~L}: 8 \mathrm{D}$ $(23,9 \mathrm{~mm}$ e $1,90 \mathrm{~g})$. Por outro lado, em relação à metamorfose, os girinos submetidos ao fotoperíodo 12L:12D apresentaram o maior percentual de metamorfose do experimento com $50 \%$ da população inicial na fase de imago (estágio 43, segundo Gosner, 1960), seguido pelos que receberam fotoperíodo $8 \mathrm{~L}: 16 \mathrm{D}$, com cerca da metade deste percentual $(22,5)$. Os animais submetidos ao fotoperíodo 16L:8D apresentaram o menor percentual de metamorfose entre estes três tratamentos $(8,9)$.

A menor taxa de metamorfose observada para o tratamento que recebeu o maior período de luz (16L:8D) está de acordo com os relatos de Delgado et al. (1984) e Gutierrez et al. (1984), que observaram em Discoglossus pictus e Xenopus laevis prejuízo no desenvolvimento dos animais submetidos a longa fase de luz. Entretanto, os resultados deste experimento diferem dos encontrados por Delgado et al. (1987), que observaram desenvolvimento mais rápido em girinos do gênero Rana quando submetidos a longa fase de luz.

O pior resultado em relação aos outros tratamentos para peso e comprimento foi apresentado pelos animais submetidos aos fotoperíodos 0L:24D (21,90 mm e 1,44 g) e 14L:10D - natural ( $23 \mathrm{~mm}$ e 1,50 g), sendo este último incluído nesta categoria, devido ao menor peso dos girinos em relação aos outros tratamentos, entretanto, no que se refere à percentagem de metamorfose, este grupo de animais poderia estar enquadrado entre aqueles de desenvolvimento intermediário, uma vez que atingiram 24,2 e $22,6 \%$, respectivamente. Estas considerações reforçam as observações de Culley Jr. et al. (1977) quanto às interpretações dos resultados experimentais com girinos.

Os resultados obtidos em relação ao crescimento dos animais estão de acordo com os relatos de Filadelfi \& Castrucci (1996), que, trabalhando com Rana esculenta e Rana temporaria, observaram que a escuridão contínua inibiu o crescimento e o desenvolvimento dos girinos destas espécies.

Nas condições em que foi conduzido este trabalho, os resultados obtidos quanto ao fotoperíodo 0L:24D sobre a metamorfose discordam dos encontrados por Doetsh (1949) e Miline (1950), citados por Dodd \& Dodd (1976) e Delgado et al. (1984 e 1987), Filadelfi \& Castrucci (1996), que verificaram a ausência de metamorfose em estudos realizados com $R$. esculenta e $R$. temporaria mantidas no escuro. Por outro lado, os resultados foram seme- 
lhantes aos de Rose \& Rose (1998), que verificaram atraso na metamorfose em girinos de Xenopus laevis, e Eichler (1976), citado por Delgado et al. (1987), que não observaram influência negativa da metamorfose em $R$. pipiens mantidos na escuridão completa.

Resultados semelhantes foram relatados por Filadelfi \& Castrucci (1996), quando os animais foram privados de luz, pois a melatonina, hormônio necessário ao desencadeamento do processo metamórfico, é produzida no escuro. Segundo os mesmos autores, girinos de $R$. esculenta e $R$. temporaria, sob escuridão constante, tiveram o crescimento e desenvolvimento inibidos, enquanto fotoperíodo curto aumentou o ganho de peso e estimulou a metamorfose em larvas de $X$. laevis.

Os resultados deste experimento estão de acordo com Hoserman et al. (1976), os quais verificaram que o desempenho dos girinos de $R$. catesbeiana é altamente variável de acordo com as condições ambientais.

Foi observado que os animais submetidos ao fotoperíodo 24L:0D apresentaram desenvolvimento lento no processo de metamorfose. Este resultado difere dos encontrados por Guyétant (1964), citado por Delgado et al.(1984), os quais observaram que a luz contínua estimulou a metamorfose em $R$. temporaria.

Delgado et al. (1984) relataram que a duração da pré-metamorfose em larvas de Discoglossus pictus expostas à luz contínua foi significativamente menor em relação àquelas mantidas na escuridão contínua, concordando com o resultado obtido neste trabalho, para girinos de $R$. catesbeiana.

Tabela 3 - Porcentagem de girinos de rã-touro (Rana catesbeiana) que atingiram o clímax de metamorfose, aos 135 dias, submetidos a diferentes fotoperíodos

Table 3 - Bullfrog tadpoles (Rana catesbeiana) percentage that reached the metamorphosis climax, submitted to different photoperiods within 135 days

\begin{tabular}{lc}
\hline $\begin{array}{l}\text { Fotoperíodo } \\
\text { Photoperiod }\end{array}$ & $\begin{array}{c}\text { Clímax da metamorfose (\%) } \\
\text { Metamorphosis climax (\%) }\end{array}$ \\
\hline 12L:12D & $50,0^{\mathrm{a}}$ \\
OL: 24D & $24,2^{\mathrm{b}}$ \\
14L:10D (natural) & $22,6^{\mathrm{b}}$ \\
8L: 16D & $22,5^{\mathrm{b}}$ \\
16L: 8D & $8,9^{\mathrm{bc}}$ \\
24L:0D & $4,4^{\mathrm{c}}$ \\
\hline
\end{tabular}

Médias seguidas de letras iguais na coluna não diferem significativamente pelo teste de Duncan a $5 \%$ de probabilidade.

Averages followed by same letters in the column do not differ significantly, according to Duncan test, at $5 \%$ of probability.
Trabalhos realizados por Wright et al. (1988) demonstraram que a fase longa de luz acelerou a metamorfose em girinos de $R$. pipiens, com a administração de $\mathrm{T}_{4}$ (tiroxina). Este resultado difere daquele encontrado no presente estudo, que mostrou ser a fase longa de luz o pior tratamento para este parâmetro.

Observações sugerem que a luz representa um papel importante na regulação da taxa de metamorfose, evidenciando uma ação desta pelo caminho do eixo TRH - TSH - TH (Hormônio liberador da tireotropina - hormônio estimulante da tireóide tiróide) em ambas as espécies, anura e urodela (Gutierrez et al., 1984).

O fotoperíodo 12L:12D apresentou resultados semelhantes aos encontrados por Delgado et al. (1987), quando observaram que este regime de luz proporcionou desenvolvimento mais rápido, quando comparado ao regime 24L:0D em X. laevis. Porém, os resultados diferem daqueles encontrados por Eichler (1976), citado por Delgado et al. (1987), observando que girinos de $R$. pipiens mantidos sob luz constante apresentaram tamanhos menores em relação àqueles submetidos ao fotoperíodo 12L:12D.

Wright et al. (1988) verificaram que a metamorfose ocorreu mais rapidamente em girinos de $R$. pipiens mantidos na fase longa de luz 12L:3D:1L:8D, em comparação à fase curta 12L:12D, seguida da administração de $\mathrm{T}_{4}$. Embora não tenha utilizada a administração de $\mathrm{T}_{4}$, observou-se que o regime 12L:12D superou os demais tratamentos quanto à metamorfose.

Wright et al. (1997) verificaram que o aumento na sensibilidade da tireóide para TSH, durante a metamorfose, poderia ser devido ao declínio dos níveis de melatonina nos estágios finais da metamorfose. Estes achados podem sugerir que o excesso de luz prejudica a metamorfose dos girinos, uma vez que este hormônio é sintetizado no escuro (Filadelfi \& Castrucci, 1996). Portanto, esta é a possível explicação para o comportamento dos animais submetidos ao fotoperíodo 24L:0D.

\section{Conclusões}

Os resultados sugerem a necessidade de mudanças no manejo da luz durante o período de girinagem, em que a alternância pode agir cinergicamente no desenvolvimento dos animais.

Trabalhos futuros são necessários para maior elucidação dos caminhos fisiológicos influenciados pela luz e refletidos no melhor desenvolvimento dos girinos de Rana catesbeiana. 


\section{Literatura Citada}

ARRUDA SOARES, H.; FONTANELLO, D.; MANDELLI JR., J. et al. Efeito da densidade de população no ganho de peso de girinos da rã-touro (Rana catesbeiana Shaw, 1802). Boletim Instituto de Pesca, v.10 (único), p.47-51, 1983.

CULLEY JR., D.D; MEYERS, S.P.; DOUCETTE JR., A.J. A high density rearing system for larval anurans. Laboratory Animal, v.6, p.34-41, 1977.

CULLEY JR., D.D.; SOTIARIDIS, P.K. Progress and problems as societed with bullfrog tadpole diets and nutrition. In: ANNUAL DR. SCHOLL CONFERENCE ON THE NUTRITION OF CAPTIVE WILD ANIMALS, 3., Chicago, Illinois. Proceedings ... Chicago: 1983. 30p.

DELGADO, M.J.; GUTIÉRREZ, P.; ALONSO-BEDATE, M. Growth reponse of premetamorphic Rana ridibunda and Discoglussus pictus tadpoles to melatonin injections and photoperiod. Acta Embryologiae et Morphologiae Experimentalis, v.5, n.1, p.23-39, 1984.

DELGADO, M.J.; GUTIÉRREZ, P.; ALONSO-BEDATE, M. Melatonin and Photoperiod alter growth and larval development in Xenopus laevis tadpoles. Comparative Biochemistry Physiology, v.86A, n.3, p.417-421, 1987.

DODD, M.H.I.; DODD, J.M. The biology of metamorphosis. In: LOFTS, B. (Ed.) Physiology of the amphibia. New York: Academic Press, 1976. v.2. p.467-599.

EUCLYDES, R.F. Manual de utilização do programa SAEG (Sistema para análises estatísticas e genéticas). Viçosa, MG: Universidade Federal de Viçosa, 1983. 59p.

FILADELFI, A.M.C.; CASTRUCCI, A.M.L. Comparative aspects of the pineal/melatonin system of poikilothermic vertebrates. Journal of Pineal Research, v.20, p.175-186, 1996.

FONTANELLO, D.; ARRUDA SOARES, H.; MANDELLI JR., J. et al. Desenvolvimento ponderal de girinos da rã-touro (Rana catesbeiana Shaw, 1802), criados com diferentes níveis protéicos. Boletim Instituto de Pesca, v.9 (único), p.125-129, 1982.

GOSNER, K.L. A simplified table for staging anuran embryos and larvae with notes on identification. Herpetologica, v.16, p.183-190, 1960.

GROMKO, M.H.; MASON, F.S.; SMITH-GILL, S.J. Analysis of the crowding effect in Rana pipiens tadpoles. The Journal of Experimental Zoology, v.186, p.63-72, 1973.
GUTIERREZ, P.; DELGADO, M.J.; ALONSO-BEDATE, M. Influence of photoperiod and melatonin administration on growth and metamorphosis in Discoglossus pictus larvae. Comparative Biochemistry Physiology, v.79A., n.2, p.255-260, 1984.

HOFFMANN, D.F.; LEBOUTE, E. M.; SOUZA, S.M.G. Efeito da temperatura e desenvolvimento de girinos de rã-touro (Rana catesbeiana Shaw, 1802). Revista Sociedade Brasileira Zootecnia. v.18, n.6, p.557-566, 1989.

HOSERMAN, N.D.; MEIER, H.; CULLEY JR., D.D. Daily variations in the effects of disturbance on growth, fattening, and metamorphosis in the bullfrog (Rana catesbeiana) tadpole. Journal of Experimental Zoology, v.198, p.353-357, 1976.

ROSE, S.M. A feedback mechanism of growth control in tadpoles. Ecology, v.41, n.1, p.188-199, 1960.

ROSE, M.F.; ROSE, S.R. Melatonin accelerates metamorphosis in Xenopus laevis. Journal of Pineal Research, v.24, p.90-95, 1998.

SEIXAS FILHO, J.T.; MELLO, S.C.R.P.; SILVA, J.M.F. et al. Efeito de níveis de energia e proteína bruta no desempenho de girinos (Rana catesbeiana, Shaw, 1802). Revista Brasileira de Zootecnia, v.27, n.4, p.664-669, 1998.

WILBUR, H.M.; COLLINS, J.P. Ecological aspects of metamorphosis. Science, v.182, p.1305-1314, 1973.

WRIGHT, M.L.; JOREY, S.T.; BLANCHARD, L.S.; BASSO, C.A. Effect of a light pulse during the dark on photoperiodic regulation of the rate of thyroxine-induced, spontaneous, and prolactin-inhibited metamorphosis in Rana pipiens tadpoles. The Journal of Experimental Zoology, v.247, p.99-108, 1988.

WRIGHT, M.L.; PIKULA, A.; BABSKI, A.M. et al. Effect of melatonin on the response of the thyroid to thyrotropin stimulation in vitro. General and Comparative Endocrinology, v.108, p.298-305, 1997. 